\title{
Comparación de valores satelitales de irradiación solar global con datos en tierra para la República De Honduras
}

\author{
Nelson BaCA ${ }^{1}$ y Marco Flores ${ }^{2}$ \\ ${ }^{1}$ Escuela de Física - UNAH, mail: nelsonomarbaca@hotmail.com \\ ${ }^{2}$ Escuela de Física - UNAH, mail: marcoaflores@yahoo.com
}

Recibido: 28 de febrero de 2017 / Aceptado: 30 de abril de 2017

\begin{abstract}
Resumen
In this paper, the comparison of satellite values monthly means of global solar irradiation of $1.25^{\circ}$ length per $1^{\circ}$ latitude spatial resolution published by NASA and open access to data is in the land of 5 locations in the Republic of Honduras. They mean square errors which vary for different locations between $2.38 \& 77.05 \%$ for the global solar radiation, depending heavily on the topographic and climatic place inhomogeneity are obtained. The media show bias relative found for most cases an overestimation by the satellite data.
\end{abstract}

Keywords: solar irradiation, satellite estimation, Honduras

En el presente trabajo, se realiza la comparación de valores satelitales medios mensuales de irradiación solar global de $1.25^{\circ}$ de longitud por $1^{\circ}$ de latitud resolución espacial publicados por la NASA y de libre acceso, con datos en tierra de 5 localidades de la República de Honduras. Se obtienen errores medios cuadráticos que varían para las distintas localidades entre 2.38 y $77.05 \%$ para la irradiación solar global, dependiendo fuertemente de la inhomogenidad topográfica y climática del lugar. Los sesgos medios relativos encontrados muestran para la mayoría de los casos una sobre estimación por parte del dato satelital.

Palabras clave:irradiación solar, estimación satelital, Honduras

\section{INTRODUCCIÓN}

$\mathrm{H}^{\mathrm{i}}$ L aprovechamiento energético de la radiación solar como fuente de energía para diversos procesos se vuelve día a día popular en el mundo y en Honduras no es la excepción. Para el diseño y evaluación de sistemas de aprovechamiento solar es necesario analizar los niveles de radiación solar que llegan a la superficie terrestre, para lo cual es necesario contar con una red que contenga la suficiente cantidad de estaciones que permita cubrir el territorio de la República de Honduras, sin embargo, Honduras aún no cuenta con una red solarimétrica, lo que hace difícil poder contar con la información de irradiación solar y aún teniendo esta red sí su densidad es baja es necesario interpolar o extrapolar datos de las distintas estaciones para estimar el error cuadrático medio relativo RMSE \%, el sesgo medio relativo MBE \% en lugares donde no hay estaciones.

Una alternativa durante el diseño de estos sistemas es la utilización de información de origen satelital. La precisión de los datos satelitales dependerá de su resolución satelital; es decir, del tamaño del pixel que puede distinguirse en una imagen o mapa. El valor del parámetro asociado a cada pixel se obtiene promediando los valores de dicho parámetro en todos los puntos del píxel. Los valores de irradiación solar satelital cuentan con la desventaja, de proveer elevados niveles de incerteza en regiones con una acentuada variabilidad climática y topográfica.

En este artículo, se comparan los valores promedios mensuales de irradiación solar global del año 1983 al año 2005 publicados por NASA en el surface meteorology and solar energy a renewable energy resource web site (release 6.0) global data sets (disponible junto a otros parámetros de radiación en https://eosweb.larc.nasa.gov) con datos en tierra de 5 localidades del país. La resolución espacial es de $1.25^{\circ}$ longitud por $1^{\circ}$ latitud y suministran valores promedios para los meses correspondientes al período 1983-2005. Estos datos son generados usando el algoritmo de onda corta Pinker y Lazlo, los datos son tomados en el International Satellite Cloud Climatology Project DX dataset (ISCCP DX). ISCCP DX obtiene datos sobre una rejilla con tamaño efectivo de pixeles 30 por $30 \mathrm{~km}$, los datos de salida se generan en una rejilla anidada que tiene una resolución de $1^{o}$ de latitud en todo el mundo y la resolución longitudinal que van desde $1^{\circ}$ en las regiones tropicales y subtropicales a $120^{\circ}$ en los polos.

Los datos en tierra, fueron obtenidos del sistema de visualización web de datos SIAP + MICROS, el cual cuenta con datos desde el año 2014, pero se utilizo los datos correspondientes del año 2015 por ser el año con el mayor número de datos. 


\section{DATOS Y MÉTODOS}

Para la comparación de datos diarios y medios mensuales en tierra con valores satelitales de irradiación solar global $(\bar{H})$, se han utilizado 5 estaciones meteorológicas de Honduras con distintas condiciones climáticas y topográficas y que poseen la mayor cantidad de datos. Los datos fueron obtenidos del sistema de visualización web de datos SIAP+MICROS, el cual cuenta con datos de 47 estaciones meteorológicas desde el año 2014, de las cuales solo 5 estaciones poseen datos completos para el año 2015. El sistema SIAP+MICROS tiene la desventaja que sus datos solo pueden ser exportados para 7 días lo que dificultó la obtención de los datos. Las estaciones analizadas se presentan en la Tabla 1 y su ubicación se muestra en la Figura 1.

\begin{tabular}{llll}
\hline Estaciones & $\operatorname{Lat}\left({ }^{\circ}\right)$ & $\operatorname{Long}\left({ }^{o}\right)$ & Altura $(m)$ \\
\hline La Ceiba & 15.739 & -86.860 & 14 \\
Tegucigalpa & 14.058 & -87.219 & 1000 \\
$\begin{array}{l}\text { Concepción } \\
\text { Santa Rosa }\end{array}$ & 13.989 & -87.248 & 990 \\
$\begin{array}{l}\text { de Copán } \\
\text { Nueva }\end{array}$ & 14.792 & -88.8 & 1143 \\
Ocotepeque & 14.430 & -89.193 & 790 \\
\hline
\end{tabular}

Tabla 1: Ubicación de las estaciones meteorológicas analizadas.

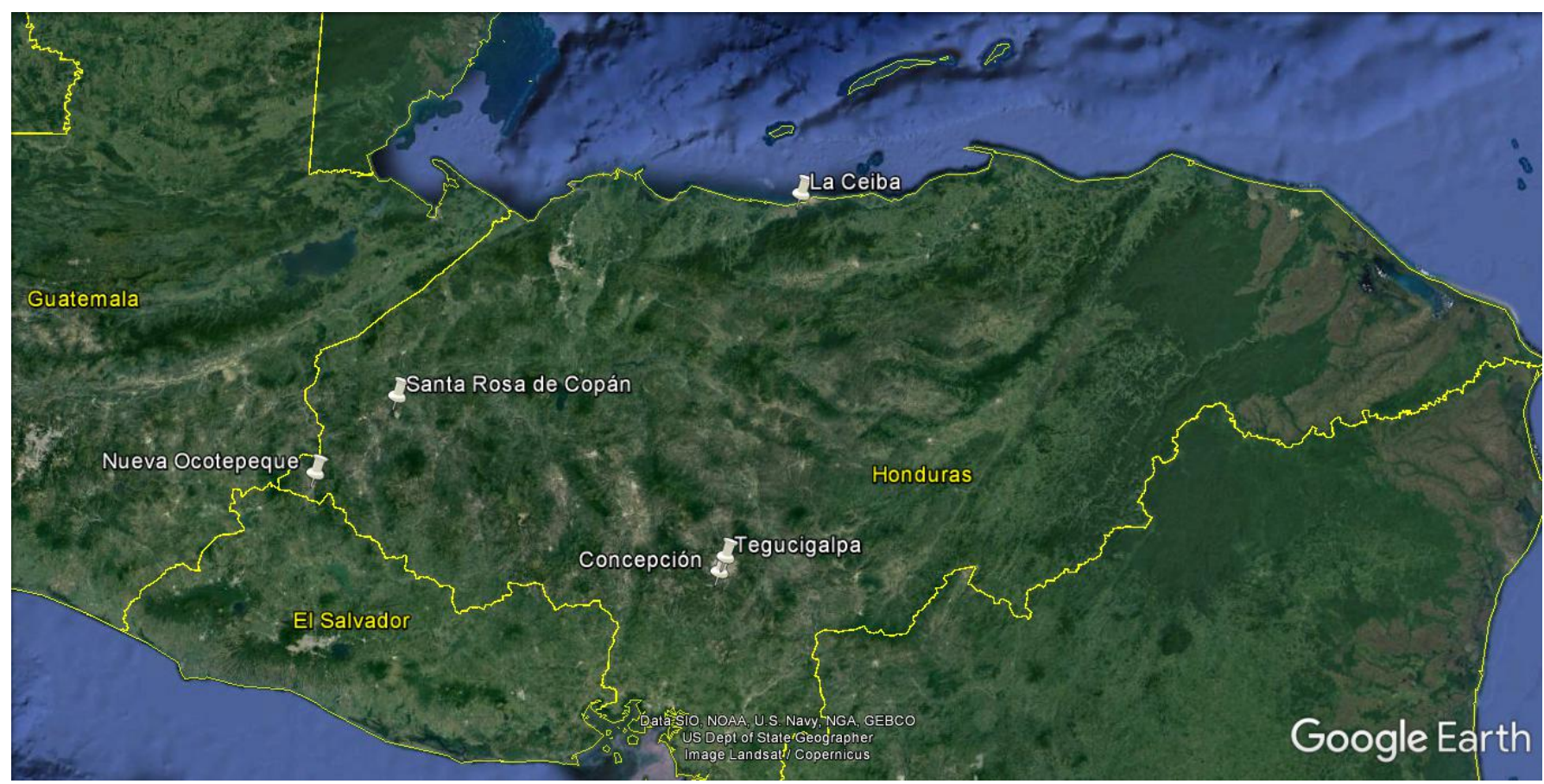

Figura 1: Ubicación de las estaciones meteorológicas analizadas

La comparación de los valores satelitales con los valores en tierra se realiza calculando la irradiación globlal $(\bar{H})$ en cada lugar y para cada mes del año los valores del error cuadrático medio relativo RMSE \% y del sesgo medio relativo $\mathrm{MBE} \%[\underline{6}$.

$$
\begin{gathered}
R M S E \%=100 * \frac{\left[\sum_{i}^{n}\left(\bar{H}_{\text {tierra }}-\bar{H}_{\text {satelital }}\right)^{2}\right]^{\frac{1}{2}}}{\sum_{i}^{n} \bar{H}_{\text {tierra }} / n} \\
M B E \%=100 * \frac{\sum_{i}^{n}\left(\bar{H}_{\text {tierra }}-\bar{H}_{\text {satelital }}\right)}{\sum_{i}^{n} \bar{H}_{\text {tierra }}}
\end{gathered}
$$

Siendo $n$ el número de casos considerados en cada mes del año.

\section{RESULTADOS Y DISCUSIÓN}

Las estaciones meteorológicas analizadas poseen clima y topografía variada. La estación meteorológica de La Ceiba se encuentra en la llanura costera atlántida con topografía muy variada desde planicies costeras como también colinas con pendientes suaves. Su clima siguiendo la clasificación de Köppen [2], es de tipo Af (clima ecuatorial) con precipitación promedio anual de $2,858 \mathrm{~mm}$, una temperatura media del mes más cálido de $27.5^{\circ} \mathrm{C}$ y una temperatura media del mes más frío de $24^{\circ} \mathrm{C}[3$. En la Tabla 2 se presentan los resultados obtenidos de los errores cuadráticos medios relativos RMSE \% y sesgos medios relativos MBE\% para la irradiación global. En la Figura 2 se muestra la comparación entre la insolación satelital y la insolación en tierra para cada mes del año. 


\begin{tabular}{lllll}
\hline Mes & $\begin{array}{l}\text { Datos en } \\
\text { tierra } \\
\left(\mathrm{kWh} / \mathrm{m}^{2} / \text { día }\right)\end{array}$ & $\begin{array}{l}\text { Datos } \\
\text { satelitales } \\
\left(\mathrm{kWh} / \mathrm{m}^{2} / \text { día }\right)\end{array}$ & RMSE \% & MBE \% \\
\hline Enero & 3.92 & 3.98 & 10.21 & -1.42 \\
Febrero & 5.97 & 4.87 & 19.83 & 18.48 \\
Marzo & 5.97 & 5.68 & 8.52 & 4.91 \\
Abril & 6.04 & 6.14 & 5.71 & -1.53 \\
Mayo & 5.70 & 5.70 & 5.36 & 0.20 \\
Junio & 5.09 & 5.63 & 12.42 & -10.50 \\
Julio & 6.27 & 5.53 & 12.97 & 11.78 \\
Agosto & 6.81 & 5.65 & 18.13 & 17.17 \\
Septiembre & 5.39 & 5.40 & 7.84 & -0.31 \\
Octubre & 4.85 & 4.43 & 2.38 & 7.93 \\
Noviembre & 4.33 & 3.82 & 14.55 & 11.01 \\
Diciembre & 4.63 & 3.66 & 22.73 & 21.07 \\
\hline
\end{tabular}

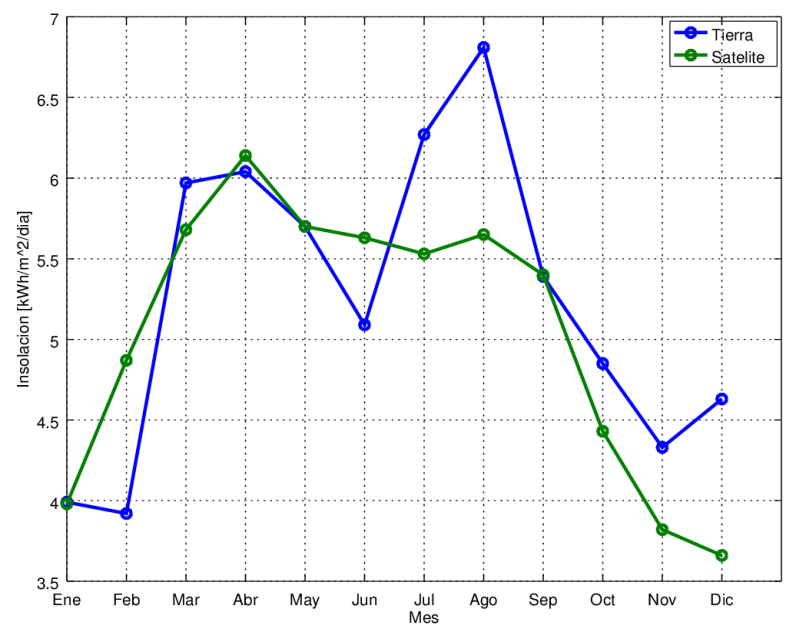

Figura 2: Comparación de insolación en La Ceiba.

Las estaciones meteorológicas de Tegucigalpa (CUUNAH) y Concepción se encuentran muy cercanas entre sí, están ubicadas en una cadena montañosa y accidentada. Se encuentran en la misma zona Af, aunque templado por la altitud, con precipitación anual promedio de $871.7 \mathrm{~mm}$, la temperatura media del mes más cálido es de $24.2^{\circ} \mathrm{C}$ y temperatura del mes mas frío de $20^{\circ} \mathrm{C}$ [2. Los resultados se consignan en la Tabla 3 y Tabla 4. La comparación de insolación se muestran en la Figura 3 y Figura 4

\begin{tabular}{lllll}
\hline Mes & $\begin{array}{l}\text { Datos en } \\
\text { tierra } \\
\left(\mathrm{kWh} / \mathrm{m}^{2} / \text { día }\right)\end{array}$ & $\begin{array}{l}\text { Datos } \\
\text { satelitales } \\
\left(\mathrm{kWh} / \mathrm{m}^{2} / \text { día }\right)\end{array}$ & RMSE \% & MBE \% \\
\hline Enero & 4.48 & 4.39 & 6.92 & 2.11 \\
Febrero & 5.63 & 5.26 & 9.94 & 6.59 \\
Marzo & 5.43 & 5.97 & 11.76 & -9.81 \\
Abril & 6.12 & 6.14 & 4.98 & -0.32 \\
Mayo & 4.84 & 5.6 & 17.05 & -15.70 \\
Junio & 4.02 & 5.48 & 36.47 & -36.06 \\
Julio & 4.90 & 5.55 & 14.76 & -13.31 \\
Agosto & 5.58 & 5.65 & 4.94 & -1.17 \\
Septiembre & 4.65 & 5.24 & 14.03 & -12.58 \\
Octubre & 4.05 & 4.60 & 18.20 & -14.41 \\
Noviembre & 4.12 & 4.29 & 8.77 & -4.31 \\
Diciembre & 4.18 & 4.14 & 7.72 & 1.24 \\
\hline
\end{tabular}

Tabla 3: Valores de RMSE \% y MBE \% para $\bar{H}$ en Tegucigalpa.

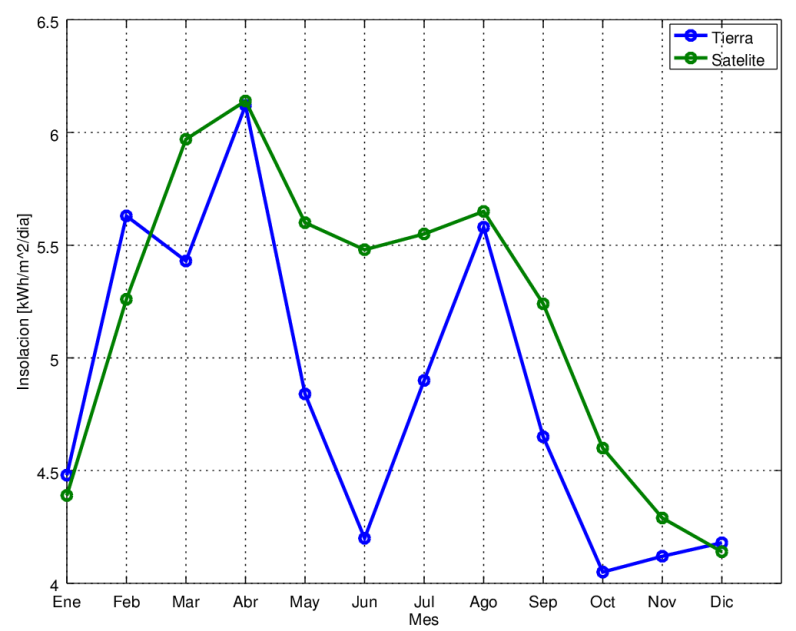

Figura 3: Comparación de insolación en Tegucigalpa.

\begin{tabular}{lllll}
\hline Mes & $\begin{array}{l}\text { Datos en } \\
\text { tierra } \\
\left(\mathrm{kWh} / \mathrm{m}^{2} / \mathrm{dia}\right)\end{array}$ & $\begin{array}{l}\text { Datos } \\
\text { satelitales } \\
\left(\mathrm{kWh} / \mathrm{m}^{2} / \text { dia }\right)\end{array}$ & RMSE $\%$ & MBE\% \\
\hline Enero & 4.98 & 5.49 & 11.24 & -10.12 \\
Febrero & 5.69 & 6.04 & 7.18 & -6.01 \\
Marzo & 5.90 & 6.45 & 10.11 & -9.26 \\
Abril & 5.72 & 6.33 & 11.89 & -10.50 \\
Mayo & 5.84 & 5.80 & 5.52 & 0.77 \\
Junio & 5.49 & 5.82 & 7.82 & -5.92 \\
Julio & 6.01 & 6.15 & 6.70 & 0.27 \\
Agosto & 6.23 & 6.0 & 7.47 & 3.75 \\
Septiembre & 5.35 & 5.31 & 8.03 & 1.07 \\
Octubre & 4.81 & 5.16 & 12.21 & -8.01 \\
Noviembre & 4.21 & 5.32 & 26.99 & -26.32 \\
Diciembre & 4.42 & 5.31 & 20.26 & -19.83 \\
\hline
\end{tabular}

Tabla 4: Valores de RMSE \% y MBE \% para $\bar{H}$ en Concepción.

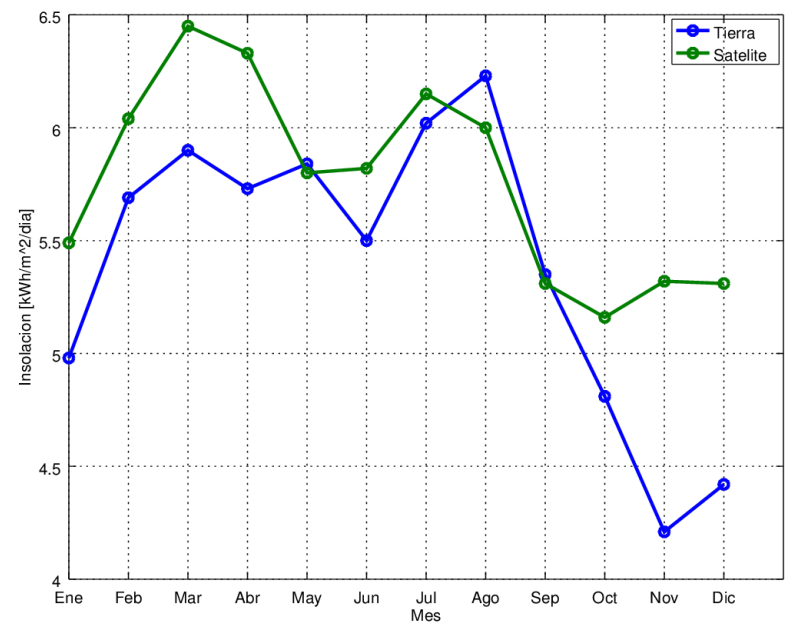

Figura 4: Comparación de insolación en Concepción.

La estación meteorológica de Santa Rosa de Copán se ubica sobre un altiplano rodeado de colinas con un clima dentro de la región Am (tropical monzónico), con 
precipitación anual promedio de 1,580.6mm, una temperatura media para el mes más cálido de $21.9^{\circ} \mathrm{C}$ y una temperatura media del mes mas frío de $17.6^{\circ} \mathrm{C}$ [4]. En la Tabla 5 se muestran los valores obtenidos de RMSE \% y MBE \%. La comparación de insolación se muestran en la Figura 5.

\begin{tabular}{lllll}
\hline Mes & $\begin{array}{l}\text { Datos en } \\
\text { tierra } \\
\left(\mathrm{kWh} / \mathrm{m}^{2} / \mathrm{dia}\right)\end{array}$ & $\begin{array}{l}\text { Datos } \\
\text { satelitales } \\
\left(\mathrm{kWh} / \mathrm{m}^{2} / \text { dia }\right)\end{array}$ & RMSE \% & MBE \% \\
\hline Enero & 3.84 & 4.60 & 21.25 & -19.67 \\
Febrero & 3.50 & 5.39 & 54.52 & -53.47 \\
Marzo & 4.90 & 6.03 & 23.53 & -23.01 \\
Abril & 5.34 & 6.22 & 17.06 & -16.28 \\
Mayo & 5.49 & 5.65 & 7.64 & -2.73 \\
Junio & 5.27 & 5.53 & 5.76 & -4.77 \\
Julio & 5.41 & 5.68 & 7.38 & -4.99 \\
Agosto & 5.59 & 5.59 & 5.40 & 0.28 \\
Septiembre & 4.15 & 5.15 & 25.21 & -23.80 \\
Octubre & 2.96 & 4.94 & 58.43 & -56.76 \\
Noviembre & 2.58 & 4.55 & 77.05 & -76.24 \\
Diciembre & 2.73 & 4.42 & 62.34 & -54.01 \\
\hline
\end{tabular}

Tabla 5: Valores de RMSE \% y MBE \% para $\bar{H}$ en Santa Rosa de Copán.

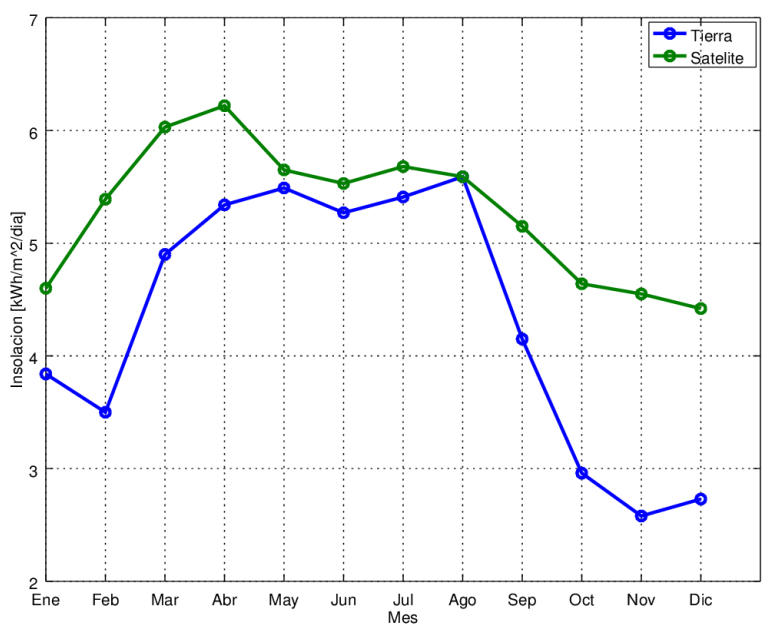

Figura 5: Comparación de insolación en Santa Rosa de Copán.

La estación meteorológica de Nueva Ocotepeque ubicada en la zona montañosa de las sierras del Merendón y Celaque se encuentra en la región Aw (tropical con invierno seco), con una precipitación anual promedio de $1,365 \mathrm{~mm}$, la temperatura media para el mes más cálido alcanza los $25^{\circ} \mathrm{C}$ y del mes más frío es de $21.1^{\circ} \mathrm{C}$ [7]. Los resultados para esta estación se muestran en la Tabla 6 y Figura 6 .

\begin{tabular}{lllll}
\hline Mes & $\begin{array}{l}\text { Datos en } \\
\text { tierra } \\
\left(\mathrm{kWh} / \mathrm{m}^{2} / \text { día }\right)\end{array}$ & $\begin{array}{l}\text { Datos } \\
\text { satelitales } \\
\left(\mathrm{kWh} / \mathrm{m}^{2} / \text { día }\right)\end{array}$ & RMSE $\%$ & MBE \% \\
\hline Enero & 5.38 & 5.03 & 8.02 & 6.53 \\
Febrero & 5.69 & 5.71 & 4.07 & -0.23 \\
Marzo & 6.28 & 6.16 & 4.56 & 2.01 \\
Abril & 6.33 & 6.26 & 5.03 & 1.20 \\
Mayo & 6.33 & 5.60 & 13.08 & 11.69 \\
Junio & 5.51 & 5.41 & 4.58 & 1.83 \\
Julio & 6.00 & 5.76 & 6.76 & 3.85 \\
Agosto & 5.97 & 5.67 & 7.63 & -5.01 \\
Septiembre & 5.43 & 5.07 & 9.15 & 6.83 \\
Octubre & 5.08 & 4.89 & 6.85 & 1.33 \\
Noviembre & 4.98 & 4.93 & 5.82 & 3.06 \\
Diciembre & 5.08 & 4.80 & 5.97 & 3.82 \\
\hline
\end{tabular}

Tabla 6: Valores de RMSE\% y MBE\% para $\bar{H}$ en Nueva Ocotepeque.

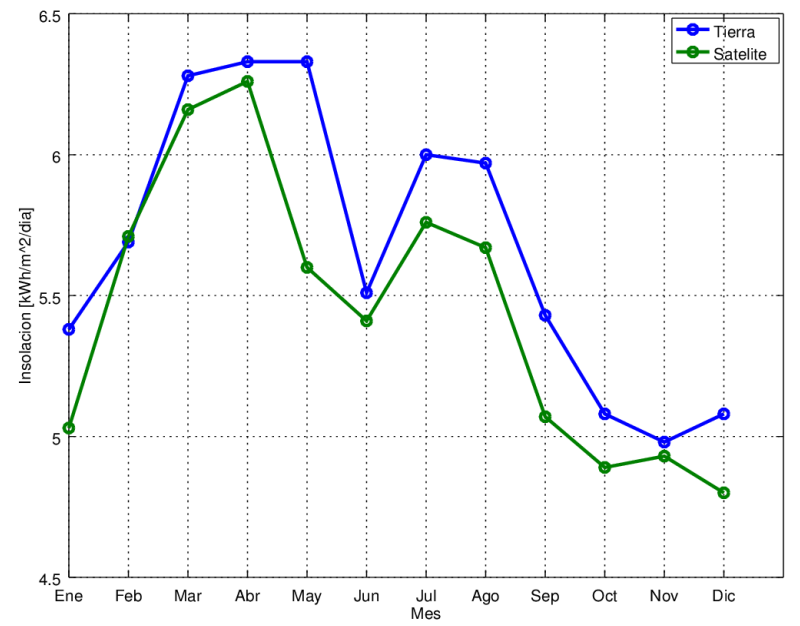

Figura 6: Comparación de insolación en Nuevo Ocotepeque.

El sistema de visualización web SIAP + MICROS solo cuenta con datos a partir del año 2014 y la NASA ha publicado datos de 1983 al 2005 lo que imposibilita la comparación simultánea. La muestra de datos utilizada en la comparación es pequeña lo cual produce un alto nivel de error en la comparación de irradiación solar global. Un factor más a considerar son los fenómenos meteorológicos, en el aõo 2015 ocurrió una alta actividad ciclónica, por lo cual la irradiación solar para los meses de septiembre a noviembre concerniente al invierno de postrera son menores que los valores satelitales.

\section{CONCLUSIONES}

La comparación de valores satelitales de irradiación solar global del año 1983 al año 2005 con datos en tierra del año 2015, muestran que los valores satelitales son un buen complemento de información para zonas en donde la temperatura no cambia bruscamente, como en Nueva Ocotepeque (Figura 6). Los valores satelitales tienen mayor error en lugares donde hay precipitaciones elevadas e inhomogenidad climática y topográfica, como tal es el caso del resto de estaciones meteorológicas analizadas. Los sesgos medios relativos muestran que en la mayoría 
de los casos existe una sobre estimación de la irradiación solar global por parte del valor satelital respecto al dato en tierra.

\section{REFERENCIAS}

[1] Google (2017), Data sio, noaa, u.s. navy, nga, gebco. google earth(versión 7.1.8.3036).

[2] Kottek, M.; Corieser, J.; Beck, C.; Rudolf, B. y Rubel, F. a. (2006). World map of the koppen-geiger climate classification updated. Meteorologische Zeitschrift, 15(3), 259-263.

[3] Merkel, A. (2013), Climate-data.org. Recuperado de: http://es.climate-data.org/location/ $879389 /$.
[4] Observatory, H. K. (2012), Climatological normals of santa rosa. Recuperado de: http: //www.weather.gov.hk/wxinfo/climat/world/ eng/s_america/mx_cam/santa_rosa_e.htm.

[5] Organization, W. M. (2012), World weather information service. Recuperado de: http://worldweather wmo. int/en/city.html?cityId=283.

[6] Raichijk, C. (2009). Comparación de valores satelitales de irradiación solar global con datos en tierra en la república de argentina. ASADES (Avances en energías Renovables y Medio ambiente), 13(11), 7-10.

[7] xplorhonduras (2013), Departamento de ocotepeque. Recuperado de: http://www.xplorhonduras com/departamento-de-ocotepeque/. 